\title{
Celgene bets big on Scripps-originated autoimmunity candidate
}

In its third major acquisition in 3 months, Celgene is buying the biotech company Receptos for US\$7.2 billion. In doing so, Celgene adds two clinical-stage drug candidates for treating autoimmune diseases to its portfolio, including one - ozanimod - that originated from the US National Institutes of Health (NIH) Molecular Libraries Program (MLP). "This is a very rare example of a compound that was discovered in academia and has made it into Phase III," says Hugh Rosen, a professor at The Scripps Research Institute (TSRI) in San Diego, USA, who co-discovered ozanimod.

In 2009, Receptos licensed a series of compounds identified in the laboratories of Rosen and his colleague, Edward Roberts, from TSRI. The researchers had screened the NIH MLP collection to identify allosteric agonists of the sphingosine-1-phosphate receptor 1 (S1PR1). The MLP initiative, which was established in 2003 to provide biomedical researchers access to the large-scale screening capacity needed to identify small molecules that could be optimized into chemical probes, ended in 2012, but legacy molecules and expertise remain.

Soon it will become apparent whether the initiative has also provided the basis for ozanimod (previously known as RPC1063) to become a marketed drug. Ozanimod is currently in two Phase III trials: one in patients with relapsing-remitting multiple sclerosis, and one in patients with ulcerative colitis. The S1PRs targeted by ozanimod are broadly distributed on lymphocytes, endothelial cells and stromal cells. "Because of their multipoint interdiction of the autoimmune cascade, [S1PR modulators] have a unique ability to have an impact on a number of difficult-to-treat autoimmune diseases," says Rosen. He expects S1PR modulation to also be particularly relevant to lupus, polymyositis and dermatomyositis, and potentially in subsets of patients with other autoimmune disorders, such as rheumatoid arthritis.

S1PRs are targeted by one drug that is already approved for the treatment of relapsing-remitting multiple sclerosis fingolimod (Novartis) - and by two others that are in Phase III trials, siponimod (Novartis) and ponesimod (Actelion). In Europe, fingolimod is only approved for patients with active disease for whom interferon- $\beta$ and glatiramer acetate are not effective. Safety issues with fingolimod most notably, fatal infections with varicella zoster virus and decreases in heart rate, both of which were observed in clinical trials - mean there is room for improvement among S1PR-targeting compounds. "It looks to me like the cardiac side effects are a bit less with ozanimod," says Bernhard Hemmer, a professor at Technische Universität München, Munich, Germany, and an expert in multiple sclerosis. However, he cautions, "one has to see how the drug performs in a Phase III trial with clinical end points and an active comparator".

Two characteristics of ozanimod may give it an edge over its direct competitors in the treatment of multiple sclerosis, according to Hemmer. The first is the reportedly higher penetrance of ozanimod into the brain relative to fingolimod, which could mean that it has greater efficacy in patients with multiple sclerosis. The second is the shorter half-life of ozanimod. "If a patient has a severe infection, you can get this drug out much faster than you can fingolimod," explains Hemmer, which means that opportunistic infections could be treated more effectively.

Two other promising immune-modulating compounds are set to enter the clinic for the treatment of multiple sclerosis by 2017: daclizumab high-yield process (Biogen/AbbVie), a CD25-specific monoclonal antibody, and ocrelizumab (Roche), which decreases the number of circulating CD20-expressing B cells. In acquiring Receptos, Celgene has bet heavily that ozanimod will eventually have a place alongside these newcomers for the treatment of multiple sclerosis and other autoimmune diseases.

Megan Cully

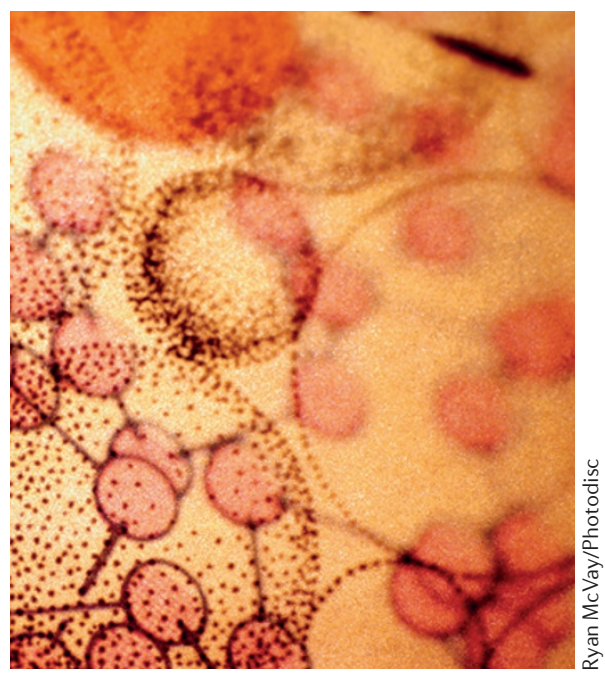

